\title{
Magnetic field and spin effects from sequential p-type and d-type triplet mechanisms $\dagger$
}

\author{
A. KATSUKI ${ }^{1}$, Y. KOBORI ${ }^{2}$, S. TERO-KUBOTA ${ }^{2}$, S. MILIKISYANTS ${ }^{3}$, \\ H. PAUL ${ }^{3}$ and U. E. STEINER ${ }^{4 *}$ \\ ${ }^{1}$ Shinshu University, Nishi-Nagano, Nagono, Japan \\ ${ }^{2}$ Tohoku University, Sendai, Japan \\ ${ }^{3}$ University of Zurich, Switzerland \\ ${ }^{4}$ Fachbereich Chemie, University of Konstanz, D-78457 Konstanz, Germany
}

\begin{abstract}
CIDEP signals of semireduced thionine radicals produced by reacting thionine triplets with aniline and halogenated anilines were measured by time resolved CW and pulsed FT EPR. For aniline as quencher, the polarization was emissive while for 4-Br- and 3-I-aniline a time dependent change in polarization from emissive to enhanced absorption was observed. For 4-I-aniline the signals were in enhanced absorption for all delay times. The time and concentration dependence of the signals was analysed in terms of a sequential double triplet mechanism: polarization of the thionine triplet due to selective population of the molecular triplet substates (classical 'p-type' triplet mechanism) and modification of this polarization by substate selective, heavy atom induced depopulation of triplet exciplexes (triplet contact radical pairs) formed as intermediates in the triplet quenching by electron transfer ('d-type' triplet mechanism). A quantitative theoretical treatment that combines the time-integrated solution of the stochastic Liouville equations for precursor triplet and triplet exciplex with the kinetic rate equation of the bimolecular quenching process is presented. The equations derived allow the extraction of two polarization enhancement factors, $V_{\mathrm{d}}$ for the pure d-type and $V_{\text {pd }}$ for the combined $\mathrm{p}$ - and d-type triplet mechanism from the concentration dependence of the time dependent CIDEP signals. The CIDEP curves and the previously observed magnetic field and heavy atom effects on the free radical yield can be quantitatively simulated with a consistent set of kinetic parameters.
\end{abstract}

\section{Introduction}

Chemically induced electron spin polarization (CIDEP) of radicals has become a powerful diagnostic tool for the study of mechanisms of radical formation and secondary chemical transformation as well as the electronic properties of the radicals and their precursors (for reviews see [1-5]). Two main types of mechanism of generation of non-Boltzmann spin-level population have been identified: the radical pair mechanism (RPM) and the triplet mechanism. Whereas the dominating interactions in the RPM comprise hyperfine coupling, exchange coupling and Zeeman interaction, spin-orbit coupling effects are the essential ingredients of the triplet mechanism. Thus, spin polarized EPR spectra due to the RPM show hyperfine dependent sign changes, whereas the CIDEP spectra due to the triplet mechanism simply show net effects of enhanced absorption or emission.

\footnotetext{
*Author for correspondence. e-mail: ulrich.steiner@unikonstanz.de

$\dagger$ Dedicated with best wishes to Professor H. G. O. Becker on the occasion of his 80th birthday.
}

The first experimental evidence of triplet-mechanismtype spin polarization was observed by Atkins et al. [6] and by Wong and Wan [7] for radicals produced from excited molecular triplets as precursors. Selective population of the zero field substates of the precursor triplet in the intramolecular intersystem crossing (ISC) following optical excitation of a singlet state was suggested as the essential cause of this spin polarization mechanism [8, 9]. It was shown that after selective population of some zero field substates in a magnetic field, the combined effect of non-vanishing zero field splitting and Zeeman splitting creates a finite magnetic polarization in the laboratory frame. This holds true even for a randomly oriented ensemble of molecules in solution. Of course, the tumbling motion of the triplet molecules in a liquid solvent acts against the polarization, first in the transfer of magnetization from the molecular frame to the laboratory frame, a process that takes about a few Larmor precession period's in time and, second, by inducing spin relaxation of the non-Boltzmann magnetization generated in the laboratory frame. Dynamic 
treatments of this mechanism have been given by Atkins and Evans [10] and by Pedersen and Freed [11].

Another aspect of the triplet mechanism was discovered by one of the present authors [12, 13] when accounting for a magnetic field effect (MFE) on the quantum yield of free radicals generated by electron transfer quenching of dye triplets by heavy atom substituted electron donors. Here the heavy atom induced spin-sublevel selective deactivation of zero field triplet substates of an intermediate exciplex, the immediate precursor of the radicals, was identified as the mechanism responsible for the MFE. Although EPR experiments were not performed with the thionine-triplet/haloaniline systems at that time, it was pointed out that the radicals emerging from the dissociation of the triplet exciplex should also be spin polarized [13]. The idea of such a mechanism had also been expressed before [14]. It is only recently that EPR experiments with these systems have been performed, using CW EPR, by the Zurich group [15] and shortly after, using pulsed FT-EPR, by the Sendai group, who had found similar effects with other systems before and coined the notion of the spin-orbit coupling mechanism (SOCM) [16-18]. However, the latter term is no longer recommended. In order to emphasize the close relationship of the original triplet mechanism that is built on selective population of zero field triplet spin sublevels and the later mechanistic variant, depending on selective depopulation of zero field spin sublevels of some radical-forming triplet precursor, the terms p-type and d-type triplet mechanism have been suggested [18]. Recently the Zurich group presented evidence that CIDEP of radicals originating from the fragmentation of a triplet is also due to the d-type triplet mechanism $[19,20]$ while the Sendai group recently also complemented their CIDEP studies in the duroquinone/halogenated $N, N$-dimethylaniline series $[18,21]$ by a flash spectroscopic investigation of the MFE on the radical yield [22] in the same systems.

The objective of the present work is to present the results of $\mathrm{CW}$ and pulsed FT EPR studies of CIDEP effects in the thionine/halogen aniline system. A unified theory based on the stochastic Liouville equation (SLE) is shown to succeed in providing a global account of both magnetic field and spin polarization effects in the spin chemistry of the thionine-triplet/haloaniline system.

\section{Experimental}

\subsection{Experiments of the Zurich group}

For the time resolved EPR measurements after flash photolytic radical generation, an Nd-YAG laser (355 nm, 6 ns pulse width) and a CW EPR detection system without field modulation ( $80 \mathrm{~ns}$ response time) were used. All further details of the experimental setup have been described previously [23].
Methanolic solutions containing thionine $\left(\left[\mathrm{TH}^{+}\right]=\right.$ $7.5 \mathrm{mM}$ ) and a substituted aniline were freed from oxygen by purging with helium and afterwards exposed to the laser irradiation while slowly flowing through a flat quartz cell $(0.7 \mathrm{~mm}$ optical path length) inside the EPR cavity. The optical density of the thionine absorption at $355 \mathrm{~nm}$ was 0.5 . The concentrations of the halogen substituted anilines were varied in the range $5-100 \mathrm{mM}$. The lower limit was given by the requirement that the quenching process had to be faster than both the time resolution of the EPR detection system and the second-order $\mathrm{T}-\mathrm{T}$ annihilation process of the thionine triplets. The flow rate of the solution was adjusted to $10 \mathrm{~cm}^{3} \mathrm{~h}^{-1}$. This corresponded to about 20 laser shots per dwell time of the irradiated volume $\left(7 \mathrm{~mm}^{3}\right)$ and kept the depletion of thionine below $5 \%$. The laser pulse energy was varied in the range $1-8 \mathrm{~mJ}$, the lower limit being determined by the detectability of the signal and the upper one by the requirement to keep the radical concentration low enough to allow a simple quantitative data analysis with neglect of second-order reaction terms in the Bloch equations. All spectra were recorded at a microwave power of $10 \mathrm{~mW}$, corresponding to a microwave field amplitude $\omega_{1} \approx 1.1 \times 10^{6} \mathrm{~s}^{-1}$ (measured from the nutation frequency of t-butyl radicals after photolysis of di-t-butyl in methanol [24]). The sensitivity factor of the spectrometer $\left(8.5 \times 10^{8} \mathrm{~V} \mathrm{~mol}^{-1}\right)$ was estimated from previous experiments [25].

All chemicals (methanol, thionine, aniline, 4-bromoaniline, 3-iodoaniline, and 4-iodoaniline) were purchased from Acros, Aldrich and Fluka in their purest available forms and were used without further purification. All measurements were carried out at room temperature.

\subsection{Experiments of the Sendai group}

Thionine chloride (Merck) was recrystallized from a mixture of ethanol, water, and conc. hydrochloric acid (50:50:1/v:v:v). Aniline (Tokyo Kasei Co.) was purified by vacuum distillation. 4-Bromoaniline and 4-iodoaniline (Merck) were recrystallized from mixtures of water and ethanol and petroleum ether. Methanol (Nacalai Co., 99.5\%) was used as solvent without purification. The concentration of thionine chloride was $2-4 \times 10^{-4} \mathrm{~mol} \mathrm{dm}^{-3}$ and that of haloanilines was $1-30 \times 10^{-3} \mathrm{~mol} \mathrm{dm}^{-3}$.

For measuring the CIDEP signals, an $\mathrm{X}$ band pulsed EPR spectrometer (Bruker ESP380E) was used. The resonator was a dielectric cavity with low $Q$ value $(\sim 100)$. The response time of the pulsed EPR instrument was estimated as $16 \mathrm{~ns}$. In order to eliminate signal distortion due to the dead time of free induction decay after a high power pulse the two-pulse echo method $(\pi / 2-\tau-\pi-\tau$-echo 
signal) was used for the measurement. An 8-step phase cycling procedure was applied for cancelling the deviation from a $90^{\circ}$ flip angle of the microwave pulse. The duration of a $\pi / 2$ pulse is estimated as $\sim 16 \mathrm{~ns}$. The second-harmonic radiation of an $\mathrm{Nd}$ :YAG laser (Spectra-Physics GCR-150, $532 \mathrm{~nm}, 30 \mathrm{~Hz}, 15 \mathrm{~mJ}$ per pulse) was used as for excitation. All sample solutions were deoxygenated by Ar gas bubbling and flowed into a quartz cell within the EPR cavity. The flow rate of the

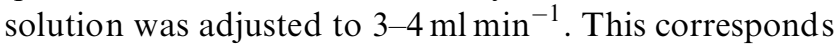
to about 5 laser shots per dwell time of the irradiated volume $\left(10 \mathrm{~mm}^{3}\right)$. All measurements were carried out at room temperature.

\section{Reaction model and theory}

The reaction model considered is presented in scheme 1. After photoexcitation to an excited singlet state, thionine undergoes fast intersystem crossing to produce the excited $\mathrm{T}_{1}$ state with a quantum yield of about 0.4 [26]. The efficiency of triplet formation in thionine contrasts with the negligible triplet formation quantum yield of oxonine, where the sulphur atom in thionine is replaced by oxygen. Thus it may be concluded that spin-orbit coupling at the sulphur center is responsible for the intersystem crossing process. The population pattern of the zero field triplet substates should therefore be governed by the local symmetry at the sulphur atom.

According to the El-Sayed rules [27, 28] direct spinorbit coupling at this centre probably will favour population of zero field substates of the triplet that have their spin orientation perpendicular to the molecular plane $\left(\mathrm{T}_{x}, \mathrm{~T}_{y}\right)$ but not with the spin in the molecular plane $\left(T_{z}\right)$. Zero field substate selective population is most

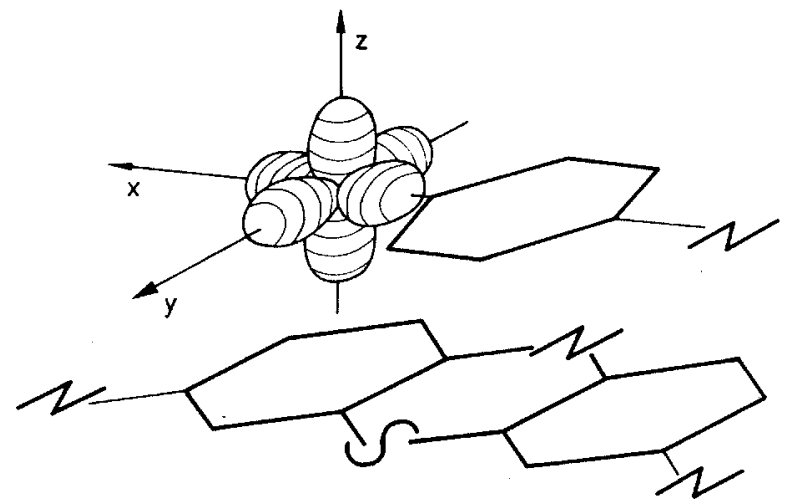

Structure 1.

likely (cf. §4) and will lead to spin polarization according to the p-type triplet mechanism. Since the triplets undergo rotational diffusion, the initial polarization will have decayed by spin relaxation to some extent before the reaction with the electron donor quencher occurs. However, the polarization present at the instant of the reaction will be transferred to the triplet exciplex (which here, for energetic reasons, has the electronic structure of a contact radical ion pair). If the electron donor moiety in the exciplex contains a heavy atom substituent, intramolecular deactivation to the singlet ground state is an efficient process that competes with exciplex dissociation into a solvent-shared radical ion pair, and finally to free radicals. The radiationless deactivation of the triplet exciplex is spin-forbidden and must be induced by spin-orbit coupling of the heavy atom substituent. Thereby another spin substate selectivity is introduced and the d-type triplet mechanism

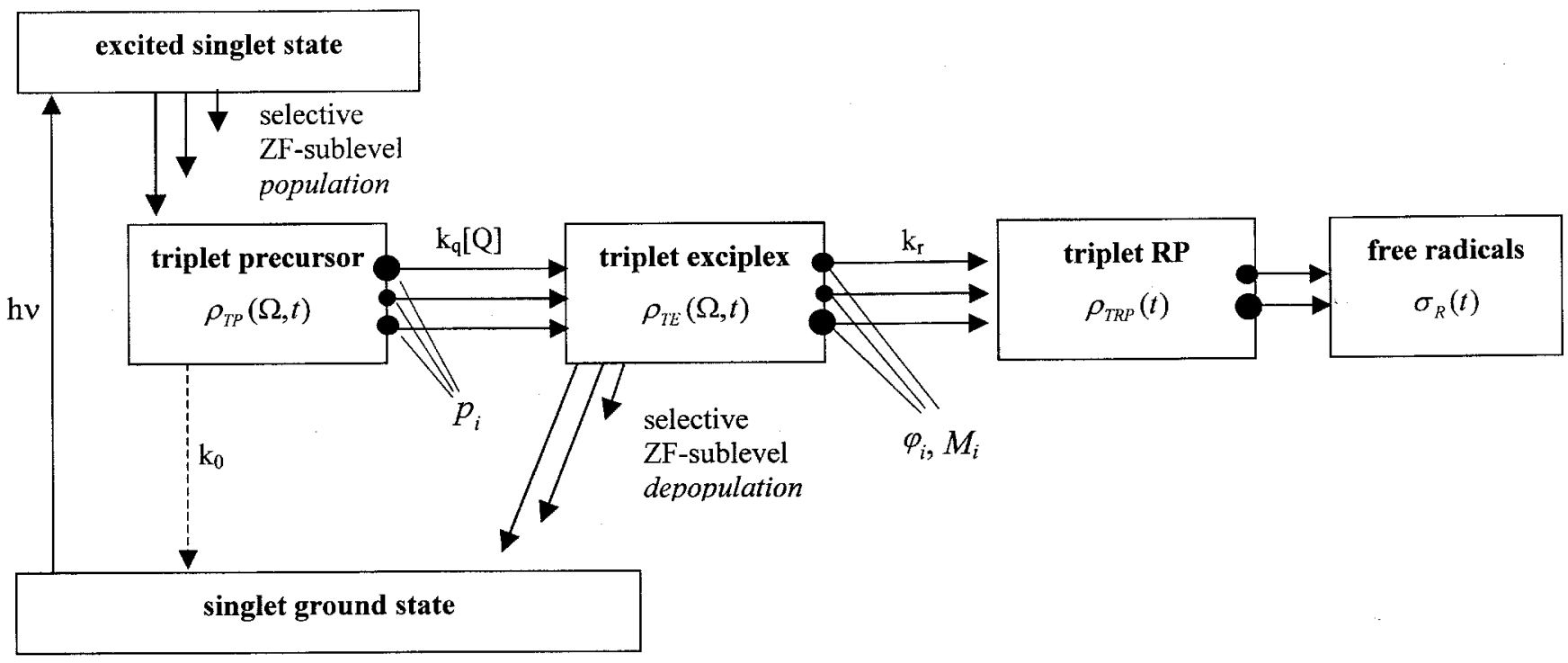

Scheme 1. 
comes into play at this stage of the reaction. The overall results to be observed experimentally are spin polarization and magnetic field dependent yield of free radicals.

The appropriate theoretical technique to deal in a quantitative way with the processes described is the spin density matrix formalism. The time dependent quantities $\rho_{\mathrm{TP}}(\Omega, t), \quad \rho_{\mathrm{TEx}}(\Omega, t), \quad \rho_{\mathrm{TRP}}(t), \sigma_{\mathrm{R}}(t)$ denote the triplet spin density matrices of the triplet precursor (TP), the triplet exciplex (TE), the solvent-share d triplet radical ion pair (TRP) and the spin density matrix of the free radicals $(\mathrm{R})$, respectively. To describe the generation of triplet-mechanism-type spin polarization, the ZFS in the triplet precursor and the triplet exciplex have to be taken into account. Thus, the spin Hamiltonians of these species becomes anisotropic, i.e. they depend on the orientation $\Omega$ of the molecular axes in the laboratory frame.

The time evolution of the spin density matrices is given by stochastic Liouville equations (SLEs) of the following general structure:

$$
\begin{aligned}
\dot{\rho}(\Omega, t)= & \mathrm{i}\left[\rho(\Omega, t), \mathbf{H}_{\mathrm{Z}}+\mathbf{H}_{\mathrm{D}}(\Omega)\right]_{-}+\Gamma_{\Omega} \rho(\Omega, t) \\
& +\frac{1}{2}[\rho(\Omega, t), \mathbf{K}(\Omega)]_{+},
\end{aligned}
$$

which has been used in a similar form by Pedersen and Freed [11] for calculating CIDEP according to the (ptype) triplet mechanism. However, the peculiar feature of the present treatment is connected with the last term of equation (1). The first term on the RHS of equation (1) describes spin motion due to the spin Hamiltonian, composed of $\mathbf{H}_{\mathrm{Z}}$, the Zeeman Hamiltonian and $\mathbf{H}_{\mathrm{D}}(\Omega)$, the ZFS Hamiltonian, that we choose as axially symmetric, so that the ZFS parameter $D$ is sufficient for its characterization. The symbol [ ] denotes the commutator. The second term describes the influence of the stochastic tumbling motion of the triplet molecules. It is given by

$$
\Gamma_{\Omega}=D_{\mathrm{r}} \nabla^{2},
$$

with $D_{\mathrm{r}}$ the rotational diffusion constant of the triplet related to the orientational correlation time $\tau_{\mathrm{r}}$ by $\tau_{\mathrm{r}}^{-1}=6 D_{\mathrm{r}}$. It should be noted that the combined effect of the first and second terms does not change the trace of the isotropically averaged spin density matrix, that is proportional to the total concentration of triplets. These terms are responsible for spin relaxation. Our treatment of this stage is restricted to the high temperature limit, i.e. it neglects the thermal polarization of the relaxed spin system. Furthermore it is assumed that the modulation of dipolar spin-spin coupling by the rotational motion of the triplet molecules (as implicit in the second term) is the dominant mechanism of spin relaxation. The third term that is distinctive for the present treatment accounts for the selective depopulation of the zero field substates of the triplet. The operator $\mathbf{K}$ is given by

$$
\mathbf{K}(\Omega)=-k_{x} \mathbf{P}_{x}(\Omega)-k_{y} \mathbf{P}_{y}(\Omega)-k_{z} \mathbf{P}_{z}(\Omega),
$$

where the $\mathbf{P}_{i}$ are projection operators onto the respective zero field substates and the $k_{i}$ are given by

$$
k_{i}=k_{\mathrm{TP}, i}+k_{\mathrm{q}}[\mathrm{Q}]
$$

for the triplet precursor and by

$$
k_{i}=k_{\mathrm{TEx}, i}+k_{\mathrm{r}}
$$

for the triplet exciplex. In the actual calculations the deactivation rate constants $k_{\mathrm{TP}, i}$ of the triplet precursor were neglected since the triplet lifetime was governed by the bimolecular quenching process. For the selectivity of heavy atom induced deactivation of the triplet exciplex, axial symmetry was assumed setting $k_{\mathrm{TEx}, x}=$ $k_{\mathrm{TEx}, y} \equiv k_{\mathrm{isc}}$ and $k_{\mathrm{TEx}, z}=0$. The anticommutator [ ] is used in order to maintain the Hermitian properties of the density matrix. It is assumed in our treatment that $\mathbf{H}_{\mathrm{D}}$ and $\mathbf{K}$ are diagonal in the same frame of molecular axes, which is probably a simplification. Equation (1) does not explicitly contain the selective population kinetics of the triplet. This aspect is taken into account in the initial conditions imposed on the solution of equation (1) (cf. [11]).

For an appropriate treatment of our kinetic problem we shall not need the full time dependent solution of $\rho_{\mathrm{TP}}(\Omega, t)$ and $\rho_{\mathrm{TEx}}(\Omega, t)$. The rise time of spin polarization in the triplet precursor is of the order of $1 / \sqrt{\omega_{0}^{2}+\tau_{\mathrm{r}}^{-2}}$. This time is on the sub-nanosecond scale, and is short on the scale of the time resolution of our experiments. On the nanosecond time scale the anisotropy of $\rho_{\mathrm{TP}}(\Omega, t)$ is negligible and we can use the usual first-order kinetic description of spin relaxation to describe the approach of the diagonal elements of the isotropically average $\rho_{\mathrm{TP}}(t)$ to thermal equilibrium.

The decay of the triplet exciplex is also very short $(\ll 1 \mathrm{~ns}[29])$ on the scale of the time resolution of our experiments. Thus in that case, too, it is sufficient to know the overall polarization created on the triplet exciplex stage and transferred to the radical stage. So we can restrict ourselves to calculating the time integrated and isotropically averaged triplet spin density matrix $\tilde{\rho}_{\mathrm{TEx}}$ defined analogously to the corresponding quantity for the triplet precursor but calculated with the appropriate parameters $D_{\mathrm{TEx}}, k_{\text {isc }}$ and $k_{\mathrm{r}}$ of the triplet exciplex.

The technique for obtaining a numerical solution for $\tilde{\rho}(\Omega)=\int_{0}^{\infty} \rho(\Omega, t) \mathrm{d} t$ from equation (1) has been described in detail by Pedersen and Freed [11]. It is based on a series expansion 


$$
\rho(\Omega, t)=\frac{1}{8 \pi^{2}} \sum_{j, m, n} \mathbf{C}_{m n}^{(j)}(t) R_{m n}^{(j)}(\Omega)
$$

of $\rho(\Omega, t)$ in terms of the Wigner functions $R_{m n}^{(j)}(\Omega)$ that form an orthonormal basis in the space of Eulerian angles $\Omega$. The time integrals of the elements $\tilde{\mathbf{C}}_{m n}^{(j)}=\int_{0}^{\infty} \mathbf{C}_{m n}^{(j)}(t) \mathrm{d} t$ of the $3 \times 3$ matrices $\mathbf{C}_{m n}^{(j)}(t)$ are obtained by numerically solving a (truncated) system of coupled linear equations. We adapted this method to include the spin-substate selective depopulation term shown in equation (1). Of the solution the quantities of interest for the present purpose are only the diagonal elements of the lowest order matrix $\tilde{\mathbf{C}}_{00}^{(0)}$ that will be denoted as follows:

$$
\begin{gathered}
\tilde{\mathbf{C}}_{00}^{(0)}(-1,-1) \equiv C_{-1}, \\
\tilde{\mathbf{C}}_{00}^{(0)}(0,0) \equiv C_{0}, \\
\tilde{\mathbf{C}}_{00}^{(0)}(1,1) \equiv C_{1} .
\end{gathered}
$$

With this information at hand we can describe the time dependence of creation of spin polarization and free radicals as follows. The SLE for the precursor triplet is solved with suitable initial conditions $\left(p_{\mathrm{T}_{x}}=p_{\mathrm{T}_{y}}=0.5, p_{\mathrm{T}_{z}}=0\right.$; for the method of treating a less selective initial population see $\S 4)$, using the known value of the ZFS of thionine $\left(D=0.069 \mathrm{~cm}^{-1}\right)$ and setting $k_{i}=k_{\text {dummy }} \ll \sqrt{\omega_{0}^{2}+\tau_{r}^{-2}}$. In the calculation $D_{\mathrm{r}}$ is set to zero and the reduction of the polarization due to suppression of polarization transfer from the molecular to the laboratory frame by rotational diffusion is accounted for by a reduction factor $r$ (a derivation of $r$ is given in the appendix). This parametrization of the SLE yields the solutions $C_{i, \mathrm{TP}}$ from which the (yet uncorrected for rotation effects) populations of the Zeeman levels of the unrelaxed p-type polarized triplet precursor are obtained as

$$
p_{i}(t=0)=k_{\text {dummy }} C_{i(\mathrm{TP})} .
$$

(Note that $\sum_{i} p_{i}=1$ ). As a consequence of longitudinal triplet spin relaxation with spin relaxation time ${ }^{3} T_{1}$ and of triplet quenching with rate constant $k_{\mathrm{q}}$, the Zeeman spin level populations of the triplet precursor decays exponentially, which is described by

$$
p_{i}(t)=\left[r\left(p_{i}(0)-p_{i, \mathrm{th}}\right) \mathrm{e}^{-t /^{3} T_{1}}+p_{i, \mathrm{th}}\right] \mathrm{e}^{-k_{\mathrm{q}}[\mathrm{Q}] t} .
$$

This approach is strictly valid only if $\left(k_{\mathrm{q}}[\mathrm{Q}]\right)^{2} \ll \omega_{0}^{2}+$ $\tau_{\mathrm{r}}^{-2}$. Note that $1 / \sqrt{\omega_{0}^{2}+\tau_{\mathrm{r}}^{-2}}$ represents the time constant for the transfer of polarization from the molecular frame to the laboratory frame. If the inequality does not hold, i.e. for very high quencher concentrations when significant quenching may occur before complete polarization transfer to the laboratory frame, a time depen- dent solution of the SLE would be needed and the monoexponential decay of $p_{i}(t)$ would have to be replaced by a more general function.

In equation (7) $r$ is the above mentioned reduction factor and $p_{i, \text { th }}$ is the population of Zeeman level $i$ of the triplet precursor in thermal equilibrium:

$$
p_{i, \mathrm{th}}=\frac{1}{3} \mathrm{e}^{-i g \beta B_{0} / k T}
$$

For simplicity it has been assumed in equation (7) that the triplet lifetime is completely determined by the bimolecular quenching process (i.e. $k_{\mathrm{q}}[\mathrm{Q}] \gg k_{0}$ ).

For the case when a triplet molecule in a definite Zeeman spin substate $i$ is quenched, the pertinent effect of the d-type triplet mechanism in the triplet exciplex is obtained from the solution of the SLE using the corresponding initial condition

$$
p_{j}(0)=\delta_{i j}
$$

and the parameters $D_{\mathrm{TEx}}, D_{\mathrm{r}}, k_{\mathrm{isc}}, k_{\mathrm{r}}$ appropriate for the triplet exciplex. This yields the set of matrix elements $C_{j(\mathrm{TE})}^{i}$ from which the radical yield $\varphi_{i}$

$$
\varphi_{i}=k_{\mathrm{r}} \sum_{j} C_{j(\mathrm{TE})}^{i}
$$

and the radical magnetization $M_{i}$ emerging from the quenching of Zeeman triplet sublevel $T_{i}^{\prime}$

$$
M_{i} \equiv 2\left\langle S_{z, i}\right\rangle[\mathrm{TP}]_{0}=k_{\mathrm{r}}\left(C_{-1(\mathrm{TE})}^{i}-C_{1(\mathrm{TE})}^{i}\right)[\mathrm{TP}]_{0}
$$

are obtained. Taking into account the time dependence of triplet decay, the following differential equations for the total yield $\varphi$ and magnetization $M$ of the radicals can be written:

$$
\dot{\varphi}(t)=k_{\mathrm{q}}[\mathrm{Q}] \sum_{i} \varphi_{i}\left[r\left(p_{i}(0)-p_{i, \mathrm{th}}\right) \mathrm{e}^{-t / T^{3} T_{1}}+p_{i, \mathrm{th}}\right] \mathrm{e}^{-k_{\mathrm{q}}[\mathrm{Q}] t},
$$

and

$$
\begin{aligned}
\dot{M}(t)= & k_{\mathrm{q}}[\mathrm{Q}] \sum_{i} M_{i}\left[r\left(p_{i}(0)-p_{i, \mathrm{th}}\right) \mathrm{e}^{-t t^{\beta} T_{1}}+p_{i, \mathrm{th}}\right] \\
& \times \mathrm{e}^{-k_{\mathrm{q}}[\mathrm{Q}] t}-\left[M(t)-\varphi(t) f_{\mathrm{B}}[\mathrm{TP}]_{0}\right]^{2} T_{1}^{-1},
\end{aligned}
$$

where $f_{\mathrm{B}}$ is the Boltzmann factor of thermal polarization of the radicals

$$
f_{\mathrm{B}}=\frac{1-\exp \left(-\frac{g \beta B_{0}}{k T}\right)}{1+\exp \left(-\frac{g \beta B_{0}}{k T}\right)} .
$$

The solution of equation (12) is 


$$
\begin{aligned}
\varphi(t)= & \frac{k_{\mathrm{q}}[\mathrm{Q}]}{1 /{ }^{3} T_{1}+k_{\mathrm{q}}[\mathrm{Q}]} \sum_{i} \varphi_{i} r\left(p_{i}(0)-p_{i, \mathrm{th}}\right) \\
& \times\left(1-\mathrm{e}^{\left.-\left(1 /{ }^{3} T_{1}+k_{\mathrm{q}}[\mathrm{Q}]\right) t\right)}\right) \\
& +\sum_{i} \varphi_{i} p_{i, \mathrm{th}}\left(1-\mathrm{e}^{-k_{\mathrm{q}}[\mathrm{Q}] t}\right),
\end{aligned}
$$

with the limiting value

$$
\begin{aligned}
\varphi \equiv \varphi(t \rightleftarrows \infty)= & \frac{k_{\mathrm{q}}[\mathrm{Q}]}{1 /{ }^{3} T_{1}+k_{\mathrm{q}}[\mathrm{Q}]} \sum_{i} \varphi_{i} p_{i}(0) \\
& +\frac{1 /{ }^{3} T_{1}}{1 /{ }^{3} T_{1}+k_{\mathrm{q}}[\mathrm{Q}]} \sum_{i} \varphi_{i} p_{i, \mathrm{th}} .
\end{aligned}
$$

The solution of equation (13) is

$$
M(t)=M_{\mathrm{th}}+A \mathrm{e}^{-t / T_{1}}+B \mathrm{e}^{-t / T_{B}}+C \mathrm{e}^{-t / T_{C}} .
$$

In order to account for the finite response time $\tau_{\exp }$ of the detection system, the result has to be convoluted with the function $\mathrm{e}^{-t / \tau_{\exp }} / \tau_{\exp }$. This transforms the terms in equation (17):

$$
\begin{aligned}
& M_{\mathrm{th}} \rightleftarrows M_{\text {th }}\left(1-\mathrm{e}^{-t / \exp }\right), \\
& \mathrm{e}^{-t / T_{n}} \rightleftarrows \frac{1}{1-\tau_{\text {exp }} / T_{n}}\left(\mathrm{e}^{-t / T_{n}}-\mathrm{e}^{-t / \tau_{\text {exp }}}\right) \text {. }
\end{aligned}
$$

The individual coefficients in equation (17) are expressed in terms of the characteristic parameters of the mechanism as follows:

$$
\begin{aligned}
M_{\mathrm{th}}= & f_{B} \varphi_{\mathrm{ce}}[\mathrm{TP}]_{0}, \\
1 / T_{B}= & k_{\mathrm{q}}[\mathrm{Q}], \\
1 / T_{C}= & 1 /{ }^{3} T_{1}+k_{\mathrm{q}}[\mathrm{Q}], \\
B= & \frac{\sum_{i} p_{i, \mathrm{th}}\left(k_{\mathrm{q}}[\mathrm{Q}] M_{i}-\varphi_{i} f_{B}[\mathrm{TP}]_{0} /{ }^{2} T_{1}\right)}{1 /{ }^{2} T_{1}-1 / T_{B}}, \\
C= & \sum_{i} r\left(p_{i}(0)-p_{i, \mathrm{th}}\right) \\
& \times \frac{\left(k_{\mathrm{q}}[\mathrm{Q}] M_{i}-\frac{k_{\mathrm{q}}[\mathrm{Q}] f_{\mathrm{B}} \varphi_{i}[\mathrm{TP}]_{0}}{\left(1 /{ }^{3} T_{1}+k_{\mathrm{q}}[\mathrm{Q}]\right)^{2} T_{1}}\right)}{1 /{ }^{2} T_{1}-1 / T_{C}}, \\
A= & -B-C-M_{\mathrm{th}} .
\end{aligned}
$$

The expressions for the coefficients $B$ and $C$ can be simplified considerably if one takes into account that, in general, the $\varphi_{i}$ differ only little. This is due to the fact that in the isotropic orientational average the molecular substates $T_{x}, T_{y}, T_{z}$ are equally represented in each of the Zeeman components $T_{i}(i=-1,0,1)$ in the laboratory frame. Hence one can assume to a good approximation that

$$
\varphi_{i} \approx \sum p_{j}(0) \varphi_{j} \approx \sum p_{t h} \varphi_{j}
$$

for all $\varphi_{i}$, and set

$$
\varphi_{i} \approx \varphi
$$

Then the limiting cases of magnetization can be expressed as:

$$
\sum p_{i, \mathrm{th}} M_{i} \equiv f_{\mathrm{B}} V_{\mathrm{d}} \varphi[\mathrm{TP}]_{0},
$$

and

$$
\sum r\left(p_{i}(0)-p_{i, \mathrm{th}}\right) M_{i}+\sum p_{i, \mathrm{th}} M_{i} \equiv f_{\mathrm{B}} V_{\mathrm{dp}} \varphi[\mathrm{TP}]_{0},
$$

where $V_{\mathrm{d}}$ represents the polarization enhancement factor of radicals due to the d-type mechanism originating from the quenching of spin-relaxed triplet precursors and $V_{\mathrm{dp}}$ represents the polarization enhancement factor of radicals due to the combined $\mathrm{d}$ - and $\mathrm{p}$-type mechanism originating from the quenching of non-relaxed p-type polarized triplet precursors. With these definitions we can rewrite the coefficients $B$ and $C$ as

$$
B=\frac{k_{\mathrm{q}}[\mathrm{Q}] V_{\mathrm{d}}-1 /{ }^{2} T_{1}}{1 /{ }^{2} T_{1}-1 / T_{B}} M_{\mathrm{th}},
$$

and

$$
C=\frac{k_{\mathrm{q}}[\mathrm{Q}]\left(V_{\mathrm{dp}}-V_{\mathrm{d}}\right)}{1 /{ }^{2} T_{1}-1 / T_{C}} M_{\mathrm{th}} .
$$

Fitting by equations (17), (18) and (19) the experimentally observed $M(t)$ curves measured relative to $M_{\text {th }}$ yields the three time constants ${ }^{2} T_{1}, T_{B}, T_{C}$ and the two (independent) coefficients $B$ and $C$. Determining them for a series of different quencher concentrations provides a data basis from which a global fit of the elementary parameters ${ }^{2} T_{1},{ }^{3} T_{1}, k_{\mathrm{q}}, V_{\mathrm{d}}$ and $V_{\mathrm{dp}}$ can be achieved.

For the analysis of the CW detected CIDEP signals it is useful to define the total radical polarization $M^{0}$ that would be obtained if spin relaxation in the radicals were negligible (i.e. ${ }^{2} T_{1} \rightleftarrows \infty$ ). This quantity can be predicted readily from the theoretical treatment here. For ${ }^{2} T_{1} \rightleftarrows \infty$, integration of equation (13) yields

$$
\begin{aligned}
M^{0} \equiv & \int_{0}^{\infty} \dot{M}(t) \mathrm{d} t=\frac{k_{\mathrm{q}}[\mathrm{Q}]}{1 /{ }^{3} T_{1}+k_{\mathrm{q}}[\mathrm{Q}]} \\
& \times\left[\sum_{i} M_{i} r\left(p_{i}(0)-p_{i, \mathrm{th}}\right)\right]+\sum_{i} M_{i} p_{i, \mathrm{th}} \\
= & \left(\frac{k_{\mathrm{q}}[\mathrm{Q}]}{1 /{ }^{3} T_{1}+k_{\mathrm{q}}[\mathrm{Q}]} V_{\mathrm{dp}}+\frac{1 /{ }^{3} T_{1}}{1 /{ }^{3} T_{1}+k_{\mathrm{q}}[\mathrm{Q}]} V_{\mathrm{d}}\right) M_{\mathrm{th}} .
\end{aligned}
$$




\section{Results}

\section{1. $C W E P R$}

After laser flash irradiation of methanolic solutions containing thionine and one of the anilines, always a nearly symmetric, almost unstructured, broad EPR line $\left(\Delta B_{1 / 2} \approx 2.3 \mathrm{mT}\right)$ was observed at $g=2.0040$, for all anilines used in this work (figure $1(a)$ ). Also, the rise and decay times of this EPR signal were very similar for all aniline compounds. The EPR time profiles differed significantly only in their maximum amplitudes, that were reached about $300 \mathrm{~ns}$ after the laser flash and indicated either emission or absorption (figure 1(b)).

We attribute the EPR signal to the semithionine radical $\left(\mathrm{TH}^{\circ}\right)$ formed with different initial spin polarizations from the triplet exciplexes ${ }^{3}\left(\mathrm{TH}^{\cdot} \mathrm{AnX}^{+\cdot}\right)$. This interpretation explains the spectral shape of the EPR signal being the same for all anilines. It is further cor-

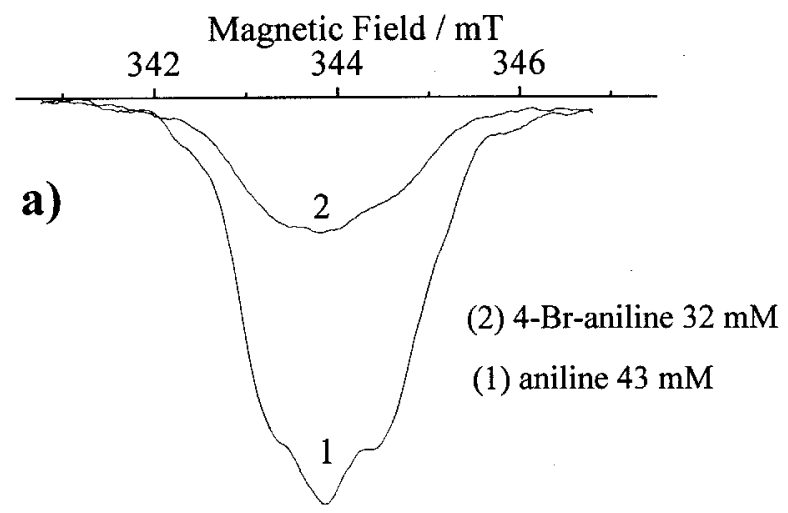

b)

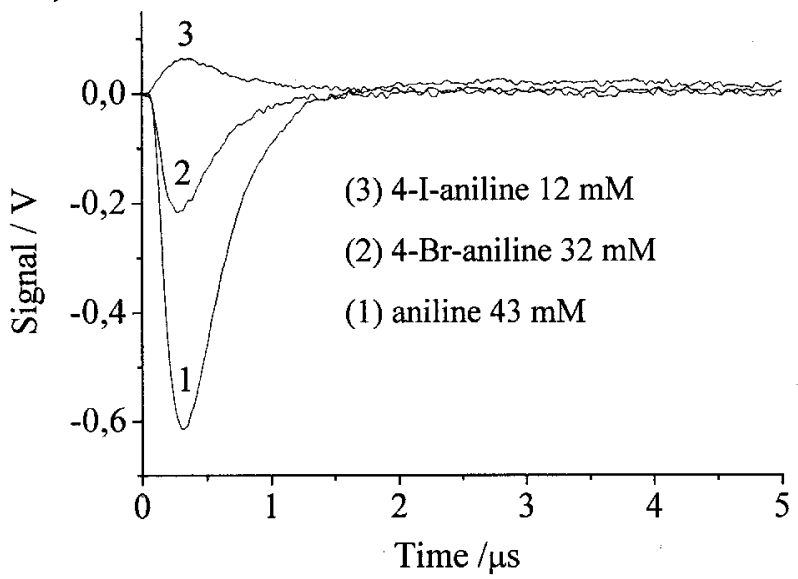

Figure 1. Time resolved CW EPR signals after flash excitation of thionine $(7.5 \mathrm{mM})$ in methanolic solutions containing various concentrations of anilines: $(a)$ magnetic field profiles $300 \mathrm{~ns}$ after the laser flash, and (b) time profiles at $3438 \mathrm{G}$. roborated by the fact that the spectral width and the $g$ factor agree well with the steady-state EPR spectrum of the thionine radical, that consists of many overlapping and only partially resolved hyperfine lines [30]. Under the conditions of the TREPR experiment, obviously the counter-radicals (the aniline cations) are EPR invisible, probably because of fast spin exchange with the aniline molecules in the solution. This finding is in line with observations made by the Sendai group (cf. also $[18,21])$.

The initial CIDEP of the thionine radical (net emission or absorption, depending on the reaction partner AnX) should be due to a p-type triplet mechanism, occurring during formation of the triplet state thionine, and a d-type TM, operative in the intersystem crossing process of the triplet exciplex back to its ground state. From the radical yields and their dependence on an applied magnetic field it is known [29] that the thionine-AnX system with $\mathrm{X}=\mathrm{p}-\mathrm{I}$ exhibits a very pronounced d-type triplet mechanism, whereas for $\mathrm{X}=\mathrm{H}$ this mechanism is negligibly small. When comparing this fact with the observed spin polarizations (figure 1(b)) one has to conclude that the d-type triplet mechanism produces an absorptive and the p-type triplet mechanism an emissive initial net CIDEP of the escaping radicals.

In principle, a third mechanism can contribute to the initial CIDEP of the radicals, namely the geminate radical pair mechanism (RPM). It would lead to a multiplet type polarization pattern, superimposed by some net CIDEP if the two species of the geminate pair have different $g$ factors. But this effect seems to be very weak in our systems, as the observed broad EPR line is symmetrical and does not indicate any noticeable multiplet polarization. Thus, we neglect any contribution from the geminate RPM.

In order to determine quantitatively the initial polarization due to $\mathrm{p}$ - and d-type triplet mechanisms, we measured the time dependence of the EPR spectra for various AnX concentrations. For an analysis of the EPR time profiles we used modified Bloch equations, describing the dynamics of the magnetization. For a single EPR transition they read

$$
\begin{aligned}
\dot{u} & =-\Delta \omega \nu-\frac{u}{{ }^{2} T_{2}}+\mathrm{O}_{1}\left([\mathrm{R}]^{2}\right), \\
\dot{\nu} & =\Delta \omega u-\frac{\nu}{{ }^{2} T_{2}}+\omega_{1} M_{z}+\mathrm{O}_{2}\left([\mathrm{R}]^{2}\right), \\
\dot{M}_{z} & =-\omega_{1} \nu-\frac{M_{z}}{{ }^{2} T_{1}}+\frac{1}{{ }^{2} T_{1}} P_{\text {eq }}[\mathrm{R}]+\mathrm{O}_{3}\left([\mathrm{R}]^{2}\right),
\end{aligned}
$$

where $\mathrm{O}_{1}, \mathrm{O}_{2}$, and $\mathrm{O}_{3}$, representing terms of second order in the radical concentration, describe the spin exchange between the radicals, their decay by bimole- 
cular termination, and the production of F-pair polarization in radical collisions [31]. The initial conditions for the equations are: $u(0)=\nu(0)=0$ and $M_{z}(0)=M_{z}^{0}$.

However, in the present case we do not measure single EPR transitions but rather the envelope of the whole thionine radical spectrum. Therefore we cannot use the above equations directly but have to integrate them over the magnetic field and then fit the result to the total experimental magnetization, i.e. the spectra, integrated over the field as well. This procedure allows us to neglect the second-order term accounting for Heisenberg spin exchange, since this exchange does not affect the total magnetization. In order to further facilitate the analysis, we have performed all measurements at low laser pulse energies, i.e. low initial radical concentrations, so that the rates of radical termination and creation of F-pair polarization remained well below the relaxation rate $\left(1 /{ }^{2} T_{1} \approx 3 \times 10^{6} \mathrm{~s}^{-1}\right)$. Under these conditions all terms in equations (33-35) that are of second order in the radical concentration may be neglected.

Laplace transformation of equations (33-35) then yields

$$
\begin{gathered}
s \tilde{u}=-\Delta \omega \tilde{\nu}-\frac{\tilde{u}}{{ }^{2} T_{2}}, \\
s \tilde{\nu}=\Delta \omega \tilde{u}-\frac{\tilde{\nu}}{{ }^{2} T_{2}}+\omega_{1} \tilde{M}_{z}, \\
s \tilde{M}_{z}=-\omega_{1} \tilde{\nu}-\frac{\tilde{M}_{z}}{{ }^{2} T_{1}}+M_{z}^{0},
\end{gathered}
$$

with the solution

$$
\tilde{\nu}=\frac{\omega_{1} M_{z}^{0}}{\left(s+\frac{1}{{ }^{2} T_{1}}\right)\left[s+\frac{1}{{ }^{2} T_{2}}+\frac{\Delta \omega^{2}}{s+\frac{1}{{ }^{2} T_{2}}}+\frac{\omega_{1}^{2}}{s+\frac{1}{{ }^{2} T_{1}}}\right]} .
$$

The measured signal $S$ is proportional to $\nu(t)$, convoluted with the response function $f(t)$ of the spectrometer, i.e.

$$
S(t)=C f(t) \otimes \nu(t),
$$

where $C$ is the sensitivity factor of the spectrometer. The response function is

$$
\left.\begin{array}{l}
t \leqslant 0, \quad f(t)=0 \\
t>0, \quad f(t)=\frac{1}{\tau_{\exp }} \mathrm{e}^{-t / \tau_{\text {exp }}}
\end{array}\right\},
$$

with $\tau_{\exp }$ being the response time of the spectrometer. The Laplace transform of the response function is $1 / 1+s \tau_{\exp }$. Hence for the Laplace transform of the signal it follows from equation (39):

$$
\tilde{S}=\frac{C \omega_{1} M_{z}^{0}}{\left(1+s \tau_{\exp }\right)\left(s+\frac{1}{{ }^{2} T_{1}}\right)\left[s+\frac{1}{{ }^{2} T_{2}}+\frac{\Delta \omega^{2}}{s+\frac{1}{2 T_{2}}}+\frac{\omega_{1}^{2}}{s+\frac{1}{{ }^{2} T_{1}}}\right]}
$$

Integration over the magnetic field then leads to

$$
\begin{aligned}
\int_{-\infty}^{\infty} \tilde{S} \mathrm{~d} \Delta \omega= & \frac{C \pi \omega_{1} M_{z}^{0}}{\left(1+s \tau_{\exp }\right)\left(s+\frac{1}{2} T_{1}\right)} \\
& \times\left[\frac{1}{1+\frac{{ }^{2} T_{1}^{2} T_{2} \omega_{1}^{2}}{\left(1+s^{2} T_{1}\right)\left(1+s^{2} T_{2}\right)}}\right]^{1 / 2}
\end{aligned}
$$

which, under the condition that $\left(\omega_{1}^{2} T_{1}^{2} T_{2}\right)^{-1} \gg 1$, can be approximated by

$$
\int_{-\infty}^{\infty} \tilde{S} \mathrm{~d} \Delta \omega \approx \frac{{ }^{2} T_{1} C \pi \omega_{1} M_{z}^{0}}{\left(1+s \tau_{\mathrm{exp}}\right)\left(1+s^{2} T_{1}\right)} .
$$

The experimental data were analysed by fitting relation (44) to the time dependent EPR spectrum after having it numerically Laplace transformed and integrated over the magnetic field. Two parameters were varied, namely $A=C \pi \omega_{1} M_{z}^{0}$ and the longitudinal relaxation rate $1 /{ }^{2} T_{1}$ of the thionine radicals. Figure 2(a) gives an example for the quality of the fits, which look reasonable in view of the various approximations implied in relation (44).

The total initial spin polarization of the radicals can be represented as

$$
M^{0}=V M_{\mathrm{th}}=V f_{\mathrm{B}}[\mathrm{R}]_{0}=V f_{\mathrm{B}} \varphi[\mathrm{TP}]_{0},
$$

where we introduced the spin polarization enhancement factor $V . M_{\mathrm{th}}$ is the magnetization of the radicals in thermal equilibrium. The total initial polarization $M^{0}$ is obtained from the fitting parameter $A$, whereby the sensitivity factor $C$ of the spectrometer for the used cell and solvent and $\omega_{1}$ were known from previous calibration experiments [25]. At low values $E_{\mathrm{abs}}$ of absorbed laser energy $M^{0}$ is proportional to $E_{\text {abs }}$ and we have

$$
M^{0}=V f_{\mathrm{B}}[\mathrm{R}]_{0}=V \frac{f_{\mathrm{B}} \phi_{\text {isc }} \varphi}{v N_{\mathrm{A}} h \nu_{\mathrm{abs}}} E_{\mathrm{abs}}
$$

where the illuminated volume $v$ has been introduced Thus from the slope of $M^{0}$ versus the absorbed laser energy (cf. figure 2(b)) and from the known values of the quantum yield $\phi_{\text {isc }}$ of 0.4 of thionine triplet formation after light absorption [32] and the quantum efficiency $\varphi$ of radical formation from the exciplex (cf. table 1) we can obtain the spin polarization enhance- 

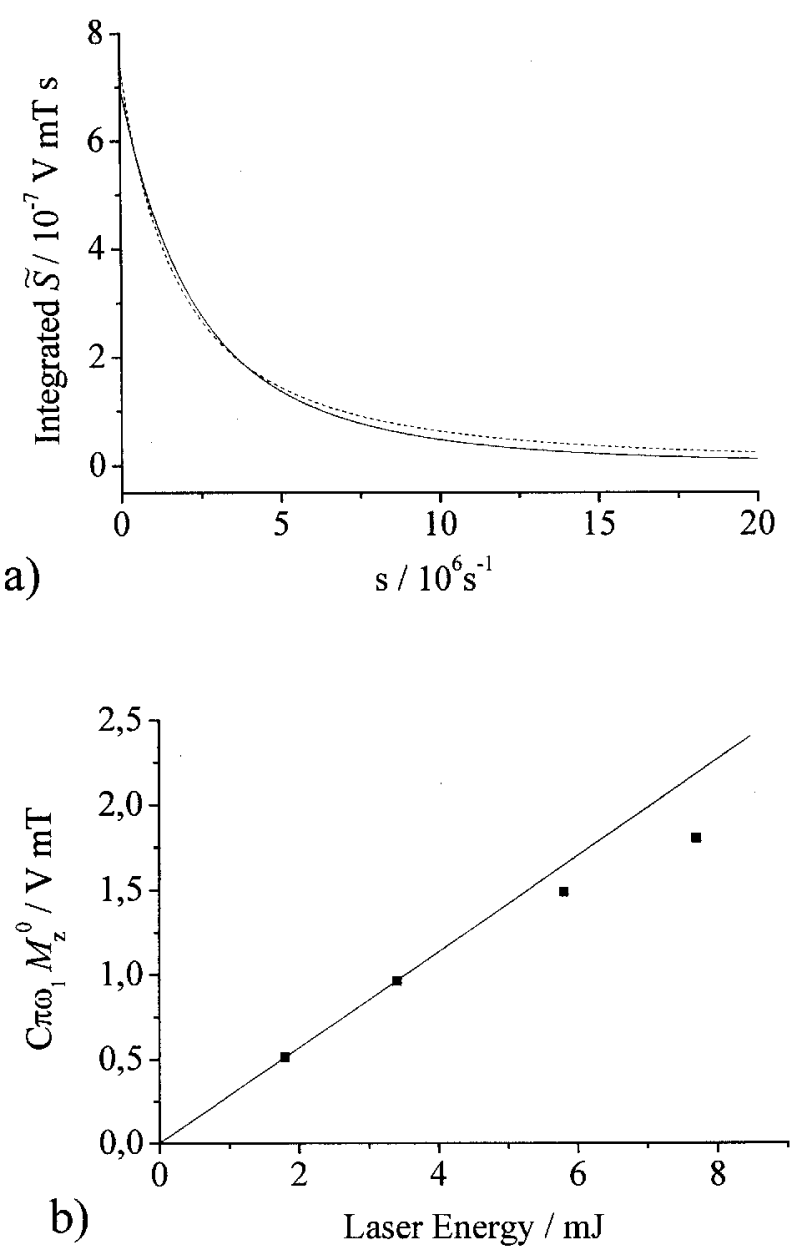

Figure 2. Determination of initial $z$ magnetization of semithionine radicals according to relations (44) and (46): (a) integrated Laplace transform of the experimental time profile (solid line) and its best fit (dotted line) with $T_{1}=380 \mathrm{~ns}$ and $C \pi \omega_{1} M_{z}^{0}=5.26 \times 10^{-7} \mathrm{VmT}$, and $(b)$ dependence of $C \pi \omega_{1} M_{z}^{0}$ on the absorbed laser pulse energy and its linear fit at low pulse energies.

ment factor $V$. This quantity has been measured at various concentrations for each of the four quenchers aniline, 4-Br-aniline, 3-I-aniline, and 4-I-aniline. The results are plotted as a function of quencher concentration in figure 3. According to equation (32) the dependence of $V$ on the quencher concentration should be described by the relation

$$
V=\frac{k_{\mathrm{q}}[\mathrm{Q}]}{1 /{ }^{3} T_{1}+k_{\mathrm{q}}[\mathrm{Q}]} V_{\mathrm{pd}}+\frac{1 /{ }^{3} T_{1}}{1 /{ }^{3} T_{1}+k_{\mathrm{q}}[\mathrm{Q}]} V_{\mathrm{d}}
$$

The lines of the corresponding fits are also shown in figure 3 , and the values of the pertinent fit parameters are given in table 1 . It should be noted, though, that the values of $k_{\mathrm{q}}$ were not treated as fit parameters but, as for

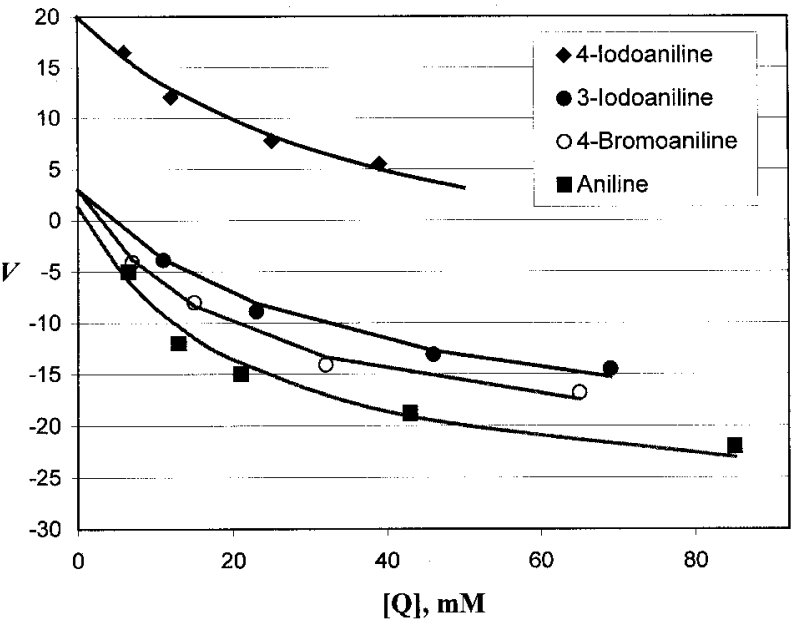

Figure 3. Dependence of total polarization enhancement factor $V$ of semithionine radicals on quencher concentration. The solid lines are the best fits with equation (47).

the values of $\varphi$, have been taken from [33], where they were determined by flash spectroscopy.

The new information obtained from the CIDEP measurements is found in the polarization enhancement factors $V_{\mathrm{d}}$ and $V_{\mathrm{pd}}$. The former, relating to the polarization enhancement of radicals that ensue from the quenching of completely spin relaxed triplets, is positive and shows a systematic trend opposite to that of the radical yield $\varphi$. The latter, relating to the polarization of radicals ensuing from the quenching of triplets, still carrying their full initial polarization from to the p-type triplet mechanism, is negative and rises in the series of halogen anilines, the trend being parallel to that seen in $V_{\mathrm{d}}$, i.e. the difference $V_{\mathrm{d}}-V_{\mathrm{pd}}$ is rather invariant (cf. table 1).

\subsection{Pulse FT-EPR}

CIDEP signals for the triplet-thionine/halogen aniline systems were also observed by pulse FT-EPR using a selection of concentrations of the quenchers aniline, 4Br-aniline, and 4-I-aniline. The time dependent magnetization $M(t)$ normalized to the final magnetization $M_{\text {th }}$, corresponding to the Boltzmann population of all radicals formed, is plotted in figure 4 . The curves observed represent the occurrence of two polarization mechanisms. For quenching by aniline, the radicals receive polarization only from the p-type triplet mechanism operating in the formation of thionine triplet by the intramolecular intersystem crossing process. This polarization is negative, as was also found in the CW EPR experiments. Again in line with the latter experiments, for quenching by 4-Br-aniline the switchover between dominant p-type polarization (at high quencher concentration) to dominant d-type polarization assigned to the 
Table 1. Kinetic parameters and polarization enhancement factors used to fit the CIDEP results obtained by $\mathrm{CW} \mathrm{EPR}^{a}{ }^{a}$

\begin{tabular}{lcccc}
\hline Quencher & Aniline & 4-Br-aniline & 3-I-aniline & 4-I-aniline \\
\hline$\varphi^{b}$ & 0.91 & 0.48 & 0.46 & 0.11 \\
${ }^{3} T_{1} / \mathrm{ns}$ & 16 & 16 & 16 & 16 \\
$k_{\mathrm{q}} / 10^{9} \mathrm{M}^{-1} \mathrm{~s}^{-1 b}$ & 3.0 & 3.0 & 1.9 & 1.6 \\
$V_{\mathrm{d}}$ & 1.3 & 3 & 3 & 20 \\
$V_{\mathrm{pd}}$ & -29 & -24 & -24 & -10 \\
$V_{\mathrm{d}}-V_{\mathrm{pd}}$ & 30.3 & 27 & 27 & 30 \\
\hline
\end{tabular}

${ }^{a}$ The values of ${ }^{2} T_{1}, \tau_{\exp }$ and $C$ used in the fits of equation (44) were the same for all systems: ${ }^{2} T_{1}=380 \mathrm{~ns}, \tau_{\exp }=100 \mathrm{~ns}, C=8.5 \times 10^{8} \mathrm{~V} \mathrm{~mol}^{-1}$.

${ }^{b}$ Values from [33].
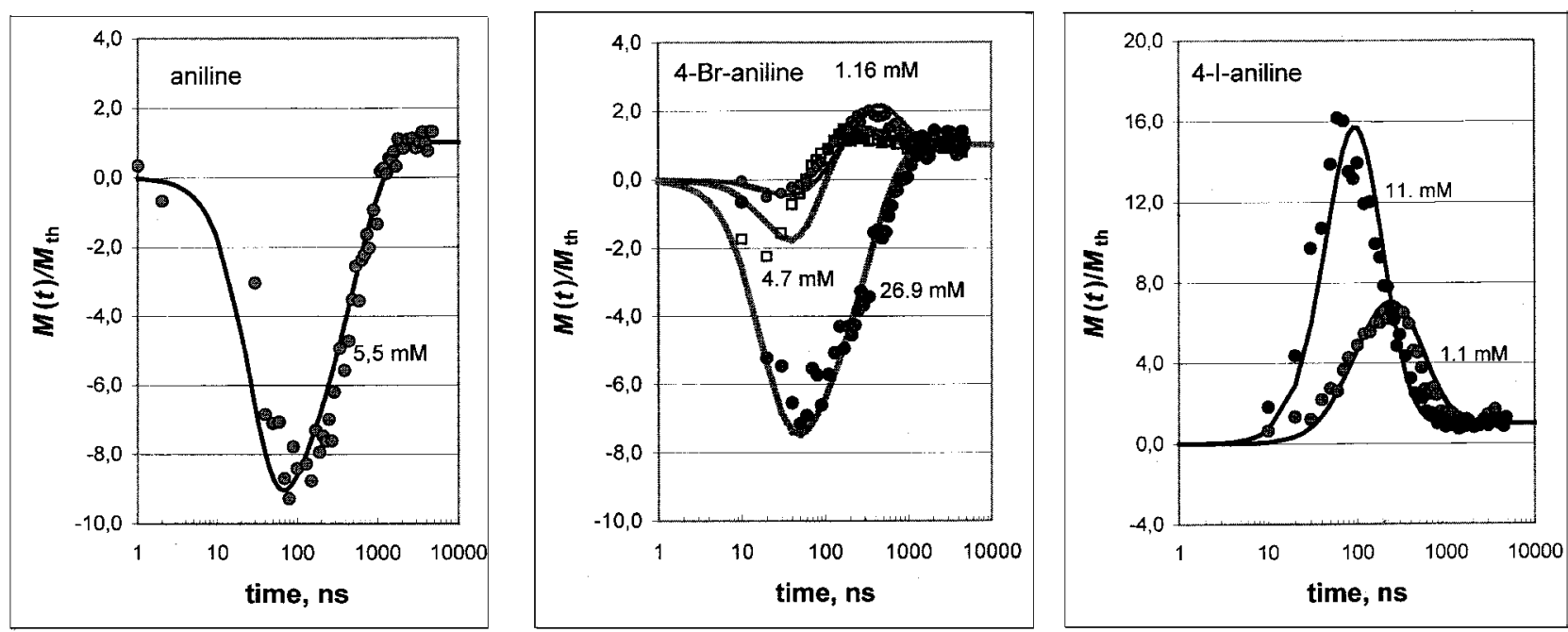

Figure 4. Time dependence of magnetization relative to final thermal equilibrium value observed by pulsed FT-EPR on quenching thionine triplet by various halogen anilines. Quencher concentrations are given on the figure. Points, experimental values; solid lines, simulations based on equations (17)-(22), (30) and (31).

partial decay by intersystem crossing of an intermediate thionine/4-Br-aniline triplet exciplex is observed. At high quencher concentrations, i.e. for such a short lifetime of the triplets that the spins cannot fully relax before the triplets are quenched, there is still exclusively negative polarization from the p-type triplet mechanism, albeit less strong than in the case of aniline. With lower concentrations there is also some negative polarization at early times but it turns to positive (larger than thermal) polarizations after several hundreds of nanoseconds. For quenching by 4-I-aniline, finally, one can only see positive polarization resulting from the d-type triplet mechanism, as was also the case for the $\mathrm{CW}$ experiments. The lowering of the signal amplitude and its shift to longer times when the 4-I-aniline concentration is only $1.1 \mathrm{mM}$ is due to the fact that with this concentration the quenching rate is smaller than the spin relaxation rate of the radicals.
As described in the theoretical part, the time dependence of $M(t)$ can be simulated using equations (17)(22), (30) and (31). The solid lines in figure 4 represent fits of the parameters of these equations to the experimental results. The pertinent fit parameters are listed in table 2. For consistence with the simulation of the results from the $\mathrm{CW}$ experiments, the longitudinal triplet spin relaxation time ${ }^{3} T_{1}$ was set to $16 \mathrm{~ns}$ for all fits. It should be noted, though, that the few concentrations employed in the pulse FT-EPR experiments are not sufficient to fix ${ }^{3} T_{1}$ unequivocally. Reasonable fits of all curves in figure 4 would be possible with even ${ }^{3} T_{1}$ as small as $3 \mathrm{~ns}$, and would lead to higher polarization enhancement factors. Such short values of ${ }^{3} T_{1}$ do not seem physically justified, however (cf. $\S 4$ ). The $k_{\mathrm{q}}$ values used in the fits correspond to the values obtained in the flash spectroscopic experiments [33] except for 4-I-aniline where a somewhat larger value of $2.5 \times 10^{9} \mathrm{M}^{-1} \mathrm{~s}^{-1}$ 
Table 2. Kinetic parameters and polarization enhancement factors used to fit the CIDEP results obtained by pulsed FT-EPR (cf. figure 4). ${ }^{a}$

\begin{tabular}{lccc}
\hline Quencher & Aniline & 4-Br-aniline & 4-I-aniline \\
\hline${ }^{3} T_{1} / \mathrm{ns}$ & 16 & 16 & 16 \\
${ }^{2} T_{1} / \mathrm{ns}$ & 400 & 300 & 120 \\
$k_{\mathrm{q}} / 10^{9} \mathrm{M}^{-1} \mathrm{~s}^{-1 b}$ & 3.0 & 3.0 & 2.5 \\
$V_{\mathrm{d}}$ & 1.3 & 6 & 37 \\
$V_{\mathrm{pd}}$ & -52 & -20 & 5 \\
$V_{\mathrm{d}}-V_{\mathrm{pd}}$ & 53.3 & 26 & 42 \\
\hline
\end{tabular}

${ }^{a}$ For the response time $\tau_{\exp }$ a value of $15 \mathrm{~ns}$ was used.

${ }^{b}$ Values from [33].

instead of $1.6 \times 10^{9} \mathrm{M}^{-1} \mathrm{~s}^{-1}$ had to be employed. Finally, it must be mentioned that the spin relaxation time ${ }^{2} T_{1}$ of the radicals had to be assumed quencherdependent, even though the signal is due mainly to the semithionine radicals in all cases.

The polarization enhancement factors $V_{\mathrm{d}}$ and $V_{\mathrm{pd}}$ obtained from the fits are also given in table 2. Their qualitative trend supports the $\mathrm{CW}$ results, but their absolute values differ from the latter. In particular, the difference $V_{\mathrm{d}}-V_{\mathrm{pd}}$ is not as constant as in the latter case.

\section{Discussion}

The present investigation of CIDEP in the thionine/ halogen aniline systems complements previous laser flash investigations dealing with the position dependent heavy atom effect [33] and MFEs on the radical yields [29] in these systems. Both CIDEP and MFEs on reaction yields are manifestations of the spin chemistry of the systems, and may be quantitatively interpreted in terms of the same mechanism (the d-type triplet mechanism) operating in the triplet contact radical ion pairs (synonymously used with 'triplet exciplexes') formed as intermediates in the quenching process. Indications of the p-type triplet mechanism cannot be seen in the MFE but only in the CIDEP signals. An overview of the relevant kinetic parameters is presented in table 3 .

The rate constants $k_{\mathrm{q}}$ of triplet quenching are not severely affected by heavy atom substitution of aniline, which is in compliance with the interpretation of the quenching as an electron transfer process, the rate of which is governed primarily by the thermodynamic driving force of the electron transfer that is hardly affected by this type of substitution [33]. On the other hand, the radical yields exhibit a systematic decrease with the spin-orbit coupling strength of the substituent, that essentially depends on two factors: its atomic spinorbit coupling constant and the spin density at the heavy atom substituent in the halogen aniline radical [33]. Thus, in 3-I-aniline the heavy atom effect, that acts to enhance the spin forbidden backward electron transfer regenerating the ground state of the pair of reactants, is less pronounced than in 4-I-aniline. This observation was the major clue for the suggestion of contactradical-pair-type triplet exciplexes as intermediates in the quenching, because in such organic radical pairs spin-orbit coupling can affect the spin only in a process where unpaired electrons change orbitals, which is feasible energetically only in the backward electron transfer itself, and requires that radicals are in close contact. (The situation would be different if the radicals had nearly degenerate electronic levels like some paramagnetic transition metal complexes (cf. [34]).) Along with the decrease in the radical yield the enhancement of spin-orbit coupling leads to an increased MFE on the free radical yield. In table 3 only one characteristic value of the relative change $R$ of $\varphi$ in a field of $1 \mathrm{~T}$ is quoted in order to characterize the MFE. For details of the magnetic field dependence see [29].

The heavy atom effect on the free radical yield and the MFE could be consistently modelled by the d-type triplet mechanism in the triplet exciplex. Thereby the triplet exciplex parameters $D_{\mathrm{r}}, k_{\mathrm{isc}}, k_{\mathrm{r}}$ i.e. rotational diffusion constant, selective intersystem crossing rate constant (assumed to occur from $T_{x}$ and $T_{y}$ only) and dissociation (cage escape) rate constant, respectively, have been assigned. From the values of these parameters it appears that the heavy atom effect on $\varphi$ and $R\left(B_{0}\right)$ is almost exclusively due to changes in the rate constant $k_{\text {isc }}$ of spin forbidden backward electron transfer. Replacing the bromine atom in 4-Br-aniline by iodine in 4-Ianiline increases $k_{\text {isc }}$ by about a factor of 7 . On the other hand, shifting the iodine atom from the 4-position to the 3 -position reduces the heavy atom effect again by about the same amount, because of the reduced spin density of the 3-position in comparison with the 4-position.

Additional characteristic quantities of the spin chemical mechanism in the thionine/halogen aniline systems are obtained from the CIDEP experiments. These are the longitudinal spin relaxation time ${ }^{3} T_{1}$ of the thionine triplet and the two polarization enhancement factors $V_{\mathrm{d}}$ and $V_{\mathrm{pd}}$. We shall focus our discussion of these quantities on the values obtained from the CW EPR experiments, since here the concentration of quenchers has been varied more extensively and the results are more consistent.

The value of $16 \mathrm{~ns}$ for ${ }^{3} T_{1}$ is based on a global fitting of the concentration dependence of the apparent polarization enhancement factors for the different anilines, whereby the experimental $k_{\mathrm{q}}$ values from the previous laser flash experiments have been used. If we apply Pedersen's and Freed's [11] theoretical result for ${ }^{3} T_{1}^{-1}$ (cf. 
Table 3. Experimental data and global set of kinetic fit parameters for magnetic field dependent kinetic laser flash spectroscopy and time resolved CIDEP.

\begin{tabular}{|c|c|c|c|c|}
\hline Quencher & Aniline & 4-Br-aniline & 3-I-aniline & 4-I-aniline \\
\hline \multicolumn{5}{|c|}{ Laser flash spectroscopy with static magnetic fields } \\
\hline$k_{\mathrm{q}} / 10^{9} \mathrm{M}^{-1} \mathrm{~s}^{-1}$ & 3.0 & 3.0 & 1.9 & 1.6 \\
\hline$\varphi$ & 0.91 & 0.48 & 0.46 & 0.11 \\
\hline$R(1 \mathrm{~T}) / \%$ & 0.0 & -1.3 & -2.3 & -20 \\
\hline \multicolumn{5}{|c|}{ (ii) Fit parameters for triplet exciplex ${ }^{b}$} \\
\hline$D_{\mathrm{r}} / 10^{9} \mathrm{~s}^{-1}$ & & 10 & 10 & 8.2 \\
\hline$k_{\text {isc }} / 10^{9} \mathrm{~s}^{-1}$ & 0 & 9.4 & 9.4 & 68 \\
\hline$k_{\mathrm{r}} / 10^{9} \mathrm{~s}^{-1}$ & & 6 & 6 & 5 \\
\hline \multicolumn{5}{|c|}{ (iii) Calculated values ${ }^{b}$} \\
\hline$\varphi$ & 1.0 & 0.50 & 0.50 & 0.135 \\
\hline$R(1 \mathrm{~T}) / \%$ & 0.0 & -1.7 & -1.7 & -21.1 \\
\hline
\end{tabular}

CIDEP

\begin{tabular}{|c|c|c|c|c|}
\hline${ }^{3} T_{1} / \mathrm{ns}$ & 16 & 16 & 16 & 16 \\
\hline$V_{\mathrm{d}}$ & 1.3 & 3 & 3 & 20 \\
\hline$V_{\mathrm{pd}}$ & -29 & -24 & -24 & -10 \\
\hline$V_{\mathrm{pd}}-V_{\mathrm{d}}$ & -30.3 & -27 & -27 & -30 \\
\hline
\end{tabular}

(ii) Fit parameters for p-type triplet mechanism in thionine and d-type triplet mechanism in

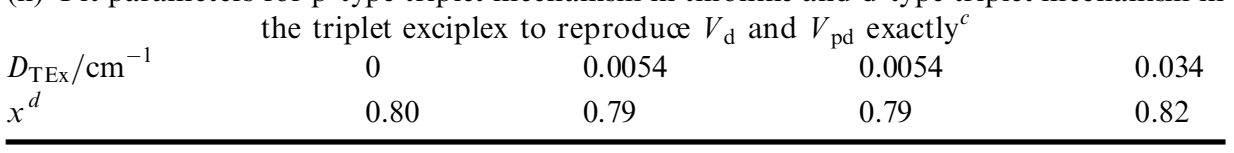

\footnotetext{
${ }^{a}$ Values from [33].

${ }^{b}$ Values from [29].

${ }^{c}$ For the parameter $D$ of thionine triplet the experimental value of 0.069 for $D^{*}$ in [35] has been used.

${ }^{d}$ The factor $x$ specifies the degree of $\mathrm{T}_{x y}$ selectivity in the intramolecular ISC process in thionine.
}

equation (A 4)) under conditions of continuous isotropic rotational diffusion and relating the orientational correlation time $\tau_{\mathrm{r}}$ to the effective molecular radius $a$ using the Debye-Stokes formula

$$
\tau_{\mathrm{r}}^{-1}=6 D_{\mathrm{r}}=\frac{3 k T}{4 \pi \eta a^{3}},
$$

we obtain $a \approx 6.8 \AA$ which seems at least a factor of 2 too large compared with the average dimension of a thionine molecule. On the other hand, the applicability of an isotropic diffusional model seems questionable for elongated planar molecules like thionine. For benzophenone, that may be considered comparable in size with thionine, a value of $6.4 \mathrm{~ns}$ for ${ }^{3} T_{1}$ has been found [20]. Since $D$ for benzophenone $\left(0.18 \mathrm{~cm}^{-1}\right.$ [36]) is larger than that for thionine, a value of $16 \mathrm{~ns}$ for the latter does not seem unreasonable.

The polarization enhancement factor $V_{\mathrm{d}}$ found for the quenching of fully spin relaxed triplets exhibits a heavy atom effect similar to that found for the sublevel selective $k_{\text {isc }}$ determined from the MFE on $\varphi$. It should be noted, though, that the value of $V_{\mathrm{d}}$ includes a contribution of $4 / 3$ which is due to a trivial thermal polarization transfer from the triplet to the radicals and, therefore, has been assigned also to aniline as quencher with which no particular enhancement of spin-orbit coupling takes place. For 4-Br-aniline and 3-I-aniline the enhancement factor $V_{\mathrm{d}}$ is found equal to within experimental error, as was the case with the $k_{\text {isc }}$ values. The slight difference in the concentration dependence of $V$ (cf. figure 3 ) can be fully attributed to the different $k_{\mathrm{q}}$ values known from laser flash spectroscopy.

Since $V_{\mathrm{d}}$ involves only one polarization step, a theoretical interpretation of it in terms of the d-type triplet mechanism is rather straightforward. In addition to the parameters already employed so far in the simulation of the MFE on $\varphi$, we introduce a specific value $D_{\mathrm{TEx}}$ for each of the exciplexes, solve the SLE and use equation 
(28) to obtain $V_{\mathrm{d}}$. With the $D_{\mathrm{TEx}}$ values presented in table 1 the experimentally determined $V_{\mathrm{d}}$ values are reproduced exactly.

To interpret the assigned $D_{\mathrm{TEx}}$ values, two contributions should be discussed: (i) the direct magnetic dipolar interaction between the unpaired electron spins and (ii) spin-orbit coupling contributions. The former may be estimated using a point-dipole approximation [37] according to which

$$
D=\frac{3}{2} \frac{g^{2} \mu_{\mathrm{B}}^{2}}{h c r^{3}},
$$

where $r$ is the distance between the two unpaired electrons and the other quantities have the usual meanings. According to this equation, a value of $D_{\mathrm{TEx}}=0.0054 \mathrm{~cm}^{-1}$ would correspond to an average distance of $7.8 \AA$. Since the electronic structure of the triplet exciplex corresponds to that of a contact radical ion pair, the two unpaired electrons are localized on two separate radical moieties and are fairly well separated. Assuming a sandwich-type geometry for the triplet exciplex, the separation of the two planes is expected to be of the order of $3.5 \AA$. Given the extension of the molecular planes of the two radicals, an average distance between the two unpaired electrons of about $8 \AA$ does not seem unreasonable. Thus it is quite likely that $D$ for the triplet exciplexes with 4-Br-aniline and 3-I-aniline is determined largely by spin dipolar interaction. Nevertheless, the d-type triplet mechanism polarization created is still a spin-orbit coupling effect, since the selective intersystem crossing is exclusively due to spin-orbit coupling. On the other hand, for 4-I-aniline, $D_{\text {Tex }}$ is much higher than reasonably expected for direct spin-spin interaction. Here the main contribution does indeed come from spin-orbit coupling, although it remains to be investigated by quantum chemical methods exactly which electronic states are involved in the coupling. From such investigations not only the assignment of the coupling states must be expected but also the assignment of the directions of the molecular axes relevant to the anisotropy of zero field splitting and of decay by selective intersystem crossing. So far, on the basis of qualitative symmetry arguments [13], we have assumed that intersystem crossing occurs selectively from $\mathrm{T}_{x}$ and $\mathrm{T}_{y}$. If this is true then the observed sign of $V_{\mathrm{d}}$ demands that $D_{\mathrm{TEx}}$ is positive.

We also want to point out that the $D_{\mathrm{TEx}}$ values obtained here are too small to be detectable in the MFE on $\varphi$. A limit of $D_{\mathrm{TEx}} \lesssim 0.05 \mathrm{~cm}^{-1}\left(\hat{=} 10 \times 10^{9} \mathrm{rad} \mathrm{s}^{-1}\right)$ had been estimated in [29].

The polarization enhancement factor $V_{\mathrm{pd}}$ characterizing the quenching of non-relaxed triplets comprises two steps of polarization: the p-type triplet mechanism in the precursor triplet followed by the d-type triplet mechanism in the triplet exciplex. Since there is no dtype triplet mechanism for aniline, the value of $V_{\mathrm{pd}}=-29$ found in this case should represent a pure p-type contribution, i.e. here $V_{\mathrm{pd}}=V_{\mathrm{p}}$. It can be shown to a first order of approximation that the overall polarization enhancement factor $V_{\text {pd }}$ of successive p-type and d-type triplet mechanism is a sum of both, viz. $V_{\mathrm{pd}} \approx V_{\mathrm{p}}+V_{\mathrm{d}}$, so that we expect $V_{\mathrm{pd}}-V_{\mathrm{d}} \approx V_{\mathrm{p}}$ to be constant. As shown in table 2, this is the case to within the accuracy of the fit of the individual $V_{\mathrm{d}}$ and $V_{\mathrm{pd}}$.

For a theoretical calculation of $V_{\text {pd }}$ by equation (29) one needs the initial Zeeman populations $p_{i}$ arising from the p-type triplet mechanism. These are obtained by solving the SLE for the decay of the precursor triplet, using the appropriate zero field splitting value $D_{\mathrm{TP}}$ and the relative population rates of the molecular zero field triplet substates as input parameters. For $D_{\mathrm{TP}}$ we can use to a good approximation the experimental value of $D^{*} \equiv \sqrt{D^{2}+3 E^{2}}=0.069 \mathrm{~cm}^{-1}$, setting $D^{*} \approx|D|$, i.e. assuming $|D / E| \gg 1$. (For example, for anthracene, which is similar in size and shape to thionine, $D=0.0718$ and $E=0.0080$, i.e. $D=0.0731$ [38]. By the analogy between thionine and anthracene we may also infer the $D$ value of the thionine triplet to be positive. The lowest substate, $\mathrm{T}_{z}$, is the one with the spin completely oriented in the aromatic plane.) The selectivity of the triplet substate population will be characterized by a fit parameter $x$ defined as

$$
1 \geqslant x \equiv \frac{k_{x}+k_{y}}{\sum k_{i}} \geq \frac{2}{3} .
$$

Since $V_{p d}$ must be a linear function of $x$ it is sufficient to solve the SLE for d-type polarization for two cases of $x$, of which $x=1$ (i.e. fully selective $\mathrm{T}_{\mathrm{xy}}$ population) and $x=2 / 3$ (unselective population of all zero field substates $\mathrm{T}_{x}, \mathrm{~T}_{y}, \mathrm{~T}_{z}$ ) leading to $p_{i}=1 / 3$ for all Zeeman sublevels, are the most convenient. Assigning to these special values of $V_{\mathrm{pd}}$ the symbols $V_{\mathrm{pd}}^{(1)}$ and $V_{\mathrm{pd}}^{(2 / 3)}$ the following general relation holds:

$$
V_{\mathrm{pd}}=3\left[(1-x) V_{\mathrm{pd}}^{(2 / 3)}-(2 / 3-x) V_{\mathrm{pd}}^{(1)}\right] .
$$

Of course, the value of $V_{\mathrm{pd}}^{(2 / 3)}$ is very similar to the value of $V_{\mathrm{d}}$, which correspond s to thermal polarization of the Zeeman levels of the precursor triplet as input condition for the d-type triplet mechanism. The difference between $V_{\mathrm{d}}$ and $V_{\mathrm{pd}}^{2 / 3}$ amounts to about $4 / 3$, and represents the transfer of thermal polarization of the triplet to the radicals. For aniline, where the exciplex is not spinorbit coupling active at all, this difference between $V_{\mathrm{d}}$ and $V_{\mathrm{pd}}^{(2 / 3)}$ is exactly $4 / 3$. For this quencher we obtain $V_{\mathrm{pd}}^{(1)}=-72.7, V_{\mathrm{d}}=4 / 3, V_{\mathrm{pd}}^{(2 / 3)}=0$. Since only a value of $V_{\mathrm{pd}}=-29$ is observed it follows from equation (51) that $x=0.80$. From the $V_{\mathrm{pd}}$ values of the other quenchers $x$ 
can be calculated in an analogous manner. As shown in table 3, all the values of $x$ range closely around 0.80 . Such an independence of $x$ on the quencher should be expected since it characterizes a quencher-independent process in the photo-excited thionine. Thus this finding corroborates the physical consistency of the parametrization. Having $x \geqslant 2 / 3$ means that in thionine the $\mathrm{T}_{x}$ and the $\mathrm{T}_{y}$ substates are preferentially (although not exclusively) populated. Since the axes $x$ and $y$ are perpendicular to the axis of the $\pi$ orbitals ( $z$ axis) this population pattern is in accord with the orbital selection rule of spin-orbit coupling if it is assumed that the sulphur atom is responsible for the intramolecular intersystem crossing process in thionine.

\section{Conclusion}

With the present investigation, the full tripletmechanism-type spin chemistry of the thionine-triplet/ halogenated aniline systems, viz. magnetic field dependence of free radical yield and time and quencher concentration dependence of CIDEP signals, has been elucidated experimentally and theoretically. The present report is one of the first examples where such a comprehensive study has been made, and the first to which the unified theoretical treatment developed here has been applied in depth. The CIDEP effects can be quantitatively accounted for by the same set of kinetic parameters explaining the magnetic field dependence of the free radical yield. They depend, however, on further parameters to which the magnetic field effect is insensitive: the longitudinal spin relaxation time of the precursor triplet, the selective population kinetics of the substates of the precursor triplet, and the ZFS parameters of the precursor triplet and the triplet exciplex. The latter becomes accessible by the present method for the first time. Although time resolved CW and pulsed FT-EPR results are in general qualitative agreement, the FT-EPR experiment, being of more preliminary nature, shows some as yet unexplained features, such as the quencher dependence of the spin relaxation time of the radicals, which should be a focus for further systematic studies.

We thank Professor B. Minaev for valuable discussions about theoretical aspects of spin-orbit coupling in triplet exciplexes. A.K. gratefully acknowledges the Japanese Ministry of Education, Culture, Sports, Science and Technology for the support of his stay as a visiting researcher at the university of Konstanz. S.M. and H.P are grateful to the Swiss National Science Foundation for Scientific Research for financial support of their part of this work.

\section{Appendix \\ Evolution of triplet spin polarization in the laboratory frame}

If the exact time dependence of magnetization $M(t)$ of the triplets in the laboratory frame after their creation with selective population of zero field molecular triplet substates at time zero were known, the total polarization of radicals obtained in a radical forming process with rate constant $k$ would be expressed as

$$
M^{0}(k)=k \int_{0}^{\infty} M(t) \mathrm{e}^{-k t} \mathrm{~d} t
$$

i.e. $M^{0}(k) / k$ can be regarded as the Laplace transform of $M(t)$ with respect to the Laplace variable $k$. In turn, the function $M(t)$ can be expressed as the inverse Laplace transform of $M^{0}(k) / k$ :

$$
M(t)=\mathcal{L}^{-1}\left(\frac{M^{0}(k)}{k}\right)
$$

For conditions justifying solution of the SLE (equation (1)) to the lowest order $(j \leqslant 2)$ of expansion in the space of the Wigner functions, the function $M^{0}(k) / k$ can be expressed in analytical form. For full axially symmetric selectivity of triplet substate population (i.e. population of $\mathrm{T}_{z}$ with probability $p_{\mathrm{T}_{z}}$ and of $\mathrm{T}_{x, y}$ with probability $p_{\mathrm{T}_{x y}}$ ) the result follows immediately from a relation given by Pedersen and Freed [11]:

$$
\begin{aligned}
\frac{M^{0}(k)}{k}= & \left(p_{\mathrm{T}_{z}}-\frac{1}{2} p_{\mathrm{T}_{x y}}\right) \frac{4 D}{15}[\mathrm{TP}]_{0} \\
& \times\left[\frac{\omega_{0}}{\omega_{0}^{2}+\tau_{\mathrm{r}}^{-2}}\left\{\frac{1}{k+{ }^{3} T_{1}^{-1}}-\frac{k+2 \tau_{\mathrm{r}}^{-1}}{\omega_{0}^{2}+\left(k+\tau_{\mathrm{r}}^{-1}\right)^{2}}\right\}\right. \\
& \left.+\frac{4 \omega_{0}}{4 \omega_{0}^{2}+\tau_{\mathrm{r}}^{-2}}\left\{\frac{1}{k+{ }^{3} T_{1}^{-1}}-\frac{k+2 \tau_{\mathrm{r}}^{-1}}{4 \omega_{0}^{2}+\left(k+\tau_{\mathrm{r}}^{-1}\right)^{2}}\right\}\right],
\end{aligned}
$$

where ${ }^{3} T_{1}^{-1}$ is given by

$$
{ }^{3} T_{1}^{-1}=\frac{2 D^{2}}{15}\left\{\frac{\tau_{\mathrm{r}}^{-1}}{\omega_{0}^{2}+\tau_{\mathrm{r}}^{-2}}+\frac{4 \tau_{\mathrm{r}}^{-1}}{4 \omega_{0}^{2}+\tau_{\mathrm{r}}^{-2}}\right\} .
$$

This approximation is valid for $D^{2} \ll \omega_{0}^{2}+\tau_{\mathrm{r}}^{-2}$. For the order of magnitude of values relevant to our systems $\left(D<0.1 \mathrm{~cm}^{-1} \hat{=} 19 \times 10^{9} \mathrm{rad} \mathrm{s}^{-1}, \quad \omega_{0} \approx 3500 \mathrm{G} \hat{=} 61 \times\right.$ $10^{9} \mathrm{rad} \mathrm{s}^{-1}$ ) the deviation from the exact numerical solution of the SLE is less than $1 \%$. By inverse Laplace transformation of equation (A 3) we obtain 


$$
\begin{aligned}
M(t)= & \left(p_{\mathrm{T}_{z}}-\frac{1}{2} p_{\mathrm{T}_{x y}}\right) \frac{4 D}{15}[\mathrm{TP}]_{0}\left[\frac{\omega_{0}}{\omega_{0}^{2}+\tau_{\mathrm{r}}^{-2}}\right. \\
& \times\left\{\mathrm{e}^{-t /^{3} T_{1}}-\left(\cos 2 \omega_{0} t+\frac{\sin \omega_{0} t}{\omega_{0} \tau_{\mathrm{r}}}\right) \mathrm{e}^{-t / \tau_{r}}\right\} \\
& +\frac{4 \omega_{0}}{4 \omega_{0}^{2}+\tau_{\mathrm{r}}^{-2}} \\
& \left.\times\left\{\mathrm{e}^{-t /^{\beta} T_{1}}-\left(\cos 2 \omega_{0} t+\frac{\sin 2 \omega_{0} t}{2 \omega_{0} \tau_{\mathrm{r}}}\right) \mathrm{e}^{-t / \tau_{r}}\right\}\right] .
\end{aligned}
$$

Considering the fact that ${ }^{3} T_{1} \gg \tau_{\mathrm{r}}$ this biexponential function describes the rise of the polarization with time constant of $\tau_{\mathrm{r}}$ (some oscillations are superimposed during this stage) and an exponential decay with time constant ${ }^{3} T_{1}$. Although equation (13) could be analytically solved with the complete expression (A 5), in our treatment (cf. equation (7)) we restrict ourselves to the monoexponential decay:

$$
\begin{aligned}
M(t)= & \left(p_{\mathrm{T}_{z}}-\frac{1}{2} p_{\mathrm{T}_{x y}}\right) \frac{4 \mathrm{D}}{15}[\mathrm{TP}]_{0} \\
& \times\left(\frac{\omega_{0}}{\omega_{0}^{2}+\tau_{\mathrm{r}}^{-2}}+\frac{4 \omega_{0}}{4 \omega_{0}^{2}+\tau_{\mathrm{r}}^{-2}}\right) \mathrm{e}^{-t /^{3} T_{1}},
\end{aligned}
$$

because the triplet quenching during the rise of polarization is negligible under the conditions of our study $\left(k_{\mathrm{q}}[\mathrm{Q}]<3 \times 10^{7} \mathrm{~s}^{-1}\right.$ whereas $\left.\tau_{\mathrm{r}}^{-1} \approx 10^{10} \mathrm{~s}^{-1}\right)$. The maximum amplitude that would ensue if $\tau_{\mathrm{r}}$ were infinity is $8\left(p_{\mathrm{T}_{z}}-\frac{1}{2} p_{\mathrm{T}_{x y}}\right)|D| / 15 \omega_{0}$. Hence the reduction factor $r$ introduced in equation (7) is given by

$$
r=\frac{1}{2}\left(\frac{\omega_{0}^{2}}{\omega_{0}^{2}+\tau_{\mathrm{r}}^{-2}}+\frac{4 \omega_{0}^{2}}{4 \omega_{0}^{2}+\tau_{r}^{-2}}\right) .
$$

For the given order of magnitude of the values of the parameters in our systems $r$ is close to 1 .

\section{References}

[1] Lepley, A. R., and Closs, G. L., 1973, Chemically Induced Magnetic Polarization (New York: Wiley).

[2] Muus, L. T., Atkins, P. W., McLauchlan, K. A., and Pedersen, J. B., 1977, Chemically Induced Magnetic Polarization (Amsterdam: Dordrecht).

[3] Salikhov, K. M., Molin, YU. M., Sagdeev, R. Z., and Buchachenko, A. L., 1984, Spin Polarization and Magnetic Effects in Radical Reactions (Amsterdam: Elsevier).

[4] Steiner, U. E., and Ulrich, T., 1989, Chem. Rev., 89, 51.

[5] Nagakura, S., Hayashi, H., and Azumi, T., 1998, Dynamic Spin Chemistry (Tokyo: Kodansha).

[6] Atkins, P. W., Buchanan, I. C., Gurd, R. C., Mclauchlan, K. A., and Simpson, A. F., 1970, Chem. Commun., 513.
[7] Wong, S. K., and Wan, J. K. S., 1972, J. Amer. chem. Soc., 94, 7197.

[8] Wong, S. K., Hutchinson, D. A., and Wan, J. K. S., 1973, J. chem. Phys., 58, 985.

[9] Atkins, P. W., and McLauchlan, K. A., 1973, Chemically Induced Magnetic Polarization, edited by A. R. Lepley and G. L. Closs (New York: Wiley Interscience) p. 41.

[10] Atrins, P. W., and Evans, G. T., 1974, Molec. Phys., 27, 1633.

[11] Pedersen, J. B., and Freed, J. H., 1974, J. chem. Phys., 62, 1706.

[12] Steiner, U. E., 1980, Chem. Phys. Lett., 74, 108.

[13] Steiner, U. E., 1981, Ber. Bunsenges. phys. Chem., 85, 228.

[14] Mclauchlan, K. A., [2], p. 151.

[15] Katsuki, A., Steiner, U. E., Milikisyants, S., and Paul, H., 1999, Book of Abstracts of the VIth International Symposium on Magnetic Field and Spin Effects in Chemistry and Related Phenomena, Emetten, p. 69.

[16] Katsuki, A., Tero-Kubota, S., and Ikegami, Y., 1993, Chem. Phys. Lett., 209, 258.

[17] Katsuki, A., Akiyama, K., Ikegami, Y., and TeroKubota, S., 1994, J. Amer. chem. Soc., 116, 12065.

[18] Sasaki, S., Katsuki, A., Akiyama, K., and TeroKubota, S., 1997, J. Amer. chem. Soc., 119, 1323.

[19] Savitsky, A. N., Batchelor, S. N., and Paul, H., 1997, Appl. magn. Reson., 13, 285.

[20] Savitsky, A. N., and Paul, H., 2000, Chem. Phys. Lett., 319, 403.

[21] Sasaki, S., Kobori, Y., Akiyama, K., and TeroKubota, S., 1998, J. phys. Chem. A, 102, 8078.

[22] Sasaki, S., Kobori, Y., Akiyama, K., and TeroKubota, S., 2001, Res. Chem. Intermed., 27, 155.

[23] Jent, F., and Paul, H., 1989, Chem. Phys. Lett., 160, 632.

[24] Savitsky, A. N., and Paul, H., 1997, Appl. magn. Reson., 12, 449.

[25] Savitsky, A. N., 1998, Ph.D. thesis, University of Zurich.

[26] Fischer, H., 1964, Z. phys. Chem. N.F., 55, 177.

[27] El-Sayed, M. A., 1963, J. chem. Phys., 38, 2834.

[28] El-Sayed, M. A., 1968, Accounts chem. Res., 1, 8.

[29] Ulrich, T., Steiner, U. E., and Föll, R. E., 1983, J. phys. Chem., 87, 1873.

[30] Schmidt, H., 1976, private communication.

[31] Savitsky, A. N., Paul, H., and Shushin, A. I., 2000, J. phys. Chem., 104, 9091.

[32] Fischer, H., 1964, Z. phys. Chem. N.F., 43, 177.

[33] Steiner, U. E., and Winter, G., 1978, Chem. Phys. Lett., 55, 364.

[34] Steiner, U. E., and Bürrner, D., 1990, Z. phys. Chem. N. F., 169, 159.

[35] Vogelmann, E., Schmidt, H., Steiner, U. E., and Kramer, H. E. A., 1975, Z. phys. Chem. N.F., 94, 101.

[36] Hochstrasser, R. M., and Lin, T.-S., 1968, J. chem. Phys., 49, 4929.

[37] Carrington, A., and Mclauchlan, A. D., 1979, Introduction to Magnetic Resonance (London: Chapman \& Hall).

[38] SixL, H., 1971, Doctoral dissertation, (University of Stuttgart. 